\title{
Anisotropic inverse conductivity and scattering problems
}

\author{
Kiwoon Kwon* Dongwoo Sheen ${ }^{\dagger}$
}

To appear in Inverse Problems, 2002

\begin{abstract}
Uniqueness in inverse conductivity and scattering problems is considered. In case the medium consists of two discontinuous constant anisotropic conductive parts, the measurements of potential and induced currents on the boundary of surrounding body are enough to guarantee uniqueness to determine conductivity and region of embedded unknown material under a very weak condition. The analogous uniqueness result is also obtained for an inverse scattering problem in the case that the medium is composed of two anisotropic and homogeneous materials.
\end{abstract}

Key words: anisotropic medium, inverse problem, conductivity, scattering

\section{Introduction}

An inverse conductivity problem, sometimes called an electrical impedance tomography problem, is to determine the interior conductivity profile or the location, shape, and size of an obstacle by measuring a pair or many pairs of current and voltage on the boundary or its part. To describe physically more rigorously, let $u$ denote the electric potential (voltage) in a bounded medium $\Omega$ with a Lipschitz boundary $\partial \Omega$ in $\mathbb{R}^{2}$ with the conductivity of the medium $\gamma=\left(\gamma_{j k}\right)_{j, k=1,2}$. Then the potential $f$ on $\partial \Omega$ and $u$ satisfies

$$
\begin{aligned}
L_{\gamma} u:=\nabla \cdot(\gamma \nabla u) & =0 \text { in } \Omega, \\
u & =f \text { on } \partial \Omega .
\end{aligned}
$$

Then current flux on $\partial \Omega$ is given by $\nu \cdot \gamma \nabla u$, where $\nu$ denotes the unit outward normal to $\Omega$.

A mathematical interpretation of an inverse conductivity problem proposed by Calderon [6] is to determine $\gamma$ from the information on the Dirichlet-to-Neumann map $\Lambda_{\gamma}: H^{1 / 2}(\Omega) \rightarrow H^{-1 / 2}(\Omega)$, It is well-known that the knowledge of $\Lambda_{\gamma}$ suffices to determine continuous, isotropic conductivity uniquely in $\mathbb{R}^{n}$ for $n=2[5,30]$, and for $n \geq 3$ [1, 29, 37, 38]. For the case of discontinuous (e.g., piecewise constant) isotropic conductivity, the question of unique determination has been considered in [4, 12, 20, 21, 22]. However, most of such results cannot be extended directly to the corresponding problem for anisotropic media, although many interesting materials are in fact anisotropic. For instance, see [25, 26, 27, 28, 36, 39] and the bibliographies therein. For the continuous conductivity case, anisotropic inverse problems have been studied in [2, 3, 27, 30, 36, 37]

\footnotetext{
${ }^{*}$ School of Mechanical and Aerospace Engineering, Seoul National University, Seoul 151-742, Korea; E-mail: kkwon@nasc.snu.ac.kr

$\dagger$ Department of Mathematics, Seoul National University, Seoul 151-747, Korea; E-mail: sheen@ snu.ac.kr
} 
For discontinuous anisotropic media, uniqueness has been known if the difference of discontinuities (or refractive indices) is positive-definite [17], and in the two dimensional case this condition is weakened so that the determinant of obstacle conductivity is different from that of background material if the conductivity matrix is small in a certain sense [16]. In this article, the smallness condition in [16] is removed in case the medium consists of two regions of homogeneous conductive materials with the determinants of conductivity of the obstacle and surrounding medium being different.

One of the popular tools in the analysis of uniqueness of inverse problems is the use of singular solutions. In what follows, we limit to anisotropic cases only. Denoting by $\Phi^{j, x}, j=2,3$, the fundamental solutions to the differential operator $L_{\gamma_{j}}$, with $x$ being the singular point, Isakov [17] observed that $\Phi^{j, x}$ satisfies the equation (2.4) in place of $u^{j}$ s. He then proceeded to induce a contradiction by showing that the left hand side in (2.4) tends to infinity as $x$ converges to a certain point, say, the origin $\mathbf{O}$. In two dimension under certain conditions, Ikehata [16] used complex functions of $O\left(\frac{1}{|x|}\right)$ growth, instead. In our paper, real functions of $O(\log |x|)$ growth are used, which have the same growth as fundamental solutions.

Our analysis can be also applied to inverse scattering problems with discontinuous anisotropic homogeneous media. which is to determine material properties (including refractive indices) or the shape and size of an obstacle by measuring scattered fields at a large distance, or equivalently measuring far-field patterns. Let $u$ and $u^{s}$ denote the scattered and total waves, respectively, generated by the incident wave $e^{i k x \cdot d}$ of wave number $k$ in the direction $d \in S^{1}$. Then the following equations hold:

$$
\begin{aligned}
L_{\gamma, k, q} u:=\nabla \cdot(\gamma \nabla u)+k^{2} q u & =0 \text { in } \mathbb{R}^{2}, \\
u(x)=e^{i k x \cdot d} & +u^{s}(x), \\
\lim _{r \rightarrow \infty} r^{\frac{1}{2}}\left(\frac{\partial u^{s}}{\partial r}-i k u^{s}\right) & =0,
\end{aligned}
$$

where $\gamma$ is the conductivity and $q$ is the refractive index in the medium. Here, $q-1$ is assumed to be an $L^{\infty}\left(\mathbb{R}^{2}\right)$ function with compact support. The far-field pattern $u_{\infty}$ then satisfies

$$
u^{s}(x)=|x|^{-\frac{1}{2}} e^{i k|x|}\left(u_{\infty}(\hat{x})+O\left(\frac{1}{|x|}\right)\right),
$$

with $\hat{x}=\frac{x}{|x|}$. An inverse scattering problem is to find $\gamma$ and $q$ where the far field patterns $u_{\infty}(\cdot ; d)$ are given for all incident direction $d \in S^{1}$ with a fixed frequency $k[7,8]$.

The theory of inverse scattering problems for isotropic media has been well-developed mathematically and numerically (see $[7,8,9,19,23,24,32,35]$ and the references therein). Corresponding theory for anisotropic media needs more attention $[10,11,14,33,34]$.

For discontinuous anisotropic media, the far-field pattern determines the medium structure uniquely provided that the jump of discontinuity is positive-definite [18]. This condition can be weakened in two dimension such that the determinants of the background and obstacle are different as far as the anisotropy near the boundary of the obstacle is known [31]. In the present paper, uniqueness is obtained without knowing the anisotropy at the boundary for discontinuous anisotropic homogeneous medium in $\mathbb{R}^{2}$.

Fundamental solutions of general elliptic partial differential and Lippmann-Schwinger-type equations are used in the analysis of inverse scattering problems [17,31], respectively. We again make use of real-valued functions of $O(\log |x|)$ which have the same type of singularity as fundamental solutions. The relation between 
the far-field operator and Dirichlet-to-Neumann map enables to apply to inverse scattering problem the analysis carried out for our inverse conductivity problem [17, 19].

We analyze in detail the inverse conductivity problem in discontinuous anisotropic media in the section to follow. Then in the subsequent section, we will apply the analysis to the inverse scattering problem of discontinuous anisotropic coefficient.

\section{Anisotropic Inverse Conductivity Problem}

\subsection{Uniqueness Result}

This section develops a uniqueness result related with the inverse problem of (1.1). Particularly, we restrict our attention to the case of the discontinuous anisotropic conductivity of the form

$$
\gamma=A+(B-A) \chi_{D}
$$

where $A$ is a known symmetric positive-definite matrix, $B$ a symmetric positive-definite matrix to be determined, and $D$ a Lipschitz domain, also to be determined, compactly contained in $\Omega \subset \mathbb{R}^{2}$ such that $\Omega \backslash \bar{D}$ is connected. Furthermore, assume that $A$ and $B$ are constant matrices.

We will then prove the following theorem.

Theorem 2.1. Let $\gamma_{j}=A+\left(B_{j}-A\right) \chi_{D_{j}}$ for $j=1,2$, where $D_{1}$ and $D_{2}$ are Lipschitz domains compactly embedded in $\Omega \subset \mathbb{R}^{2}$ such that $\Omega \backslash \bar{D}_{j}, j=1,2$ are connected, and $B_{1}$ and $B_{2}$ are $2 \times 2$ constant symmetric positive-definite matrices. If $\operatorname{det}\left(B_{j}\right) \neq \operatorname{det}(A)$, then $\Lambda_{\gamma_{1}}=\Lambda_{\gamma_{2}}$ implies

$$
D_{1}=D_{2} \text { and } B_{1}=B_{2} \text {. }
$$

\subsection{The Proof of Theorem 2.1: $D_{1}=D_{2}$}

We begin by simplifying the types of $\gamma_{j}, j=1,2$. Since $A$ is symmetric positive-definite, there exist an invertible matrix $C$ such that $A=C^{t} C$. Identify $C$ with the linear transformation $\widetilde{x} \mapsto x=C \widetilde{x}$, and denoting $\widetilde{\Omega}=C^{-1}(\Omega), \widetilde{D}=C^{-1}(D), \widetilde{f}(\widetilde{x})=f(C \widetilde{x})$, and $\widetilde{\gamma}(\widetilde{x})=C^{-t} A C^{-1}+\left(C^{-t} B C^{-1}-C^{-t} A C^{-1}\right) \chi_{\widetilde{D}}(C \widetilde{x})$. Then Problem (1.1) can be reduced to

$$
\begin{aligned}
& \nabla_{\widetilde{x}} \cdot\left(I+(\widetilde{B}-I) \chi_{\widetilde{D}}\right) \nabla_{\widetilde{x}} \widetilde{u}=0 \quad \text { in } \quad \widetilde{\Omega}, \\
& \widetilde{u}=\widetilde{f} \quad \text { on } \quad \partial \widetilde{\Omega},
\end{aligned}
$$

where $\widetilde{B}=C^{-t} B C^{-1}$ is a symmetric positive-definite matrix. Therefore, without loss of generality, the matrix $A$ may be assumed to be the identity matrix $I$ in the original equations (1.1). The tildes will be dropped from now on.

Furthermore, consider an orthogonal matrix $P$ such that $B_{1}=P^{t} \Sigma P$, where $\Sigma$ is a diagonal matrix whose diagonal elements consist of eigenvalues of $B_{1}$. Again, by the identification of $P^{-1}$ with the induced linear transformation $\widetilde{x} \mapsto x=P^{-1} \widetilde{x}$, Equations (2.3) can be reduced into a simpler setting in which $B_{1}$ is a diagonal matrix with positive diagonal entries while $B_{2}$ is a symmetric, positive-definite matrix. 
Summing up, we henceforth assume that

$$
A=I, \quad B_{1}=\left(\begin{array}{cc}
\lambda_{1} & 0 \\
0 & \lambda_{2}
\end{array}\right), \quad \lambda_{1}, \lambda_{2}>0
$$

and $B_{2}$ is a positive-definite matrix in Equations (2.3). Also, without loss of generality, we may assume

$$
\begin{cases}\lambda_{1} \leq \lambda_{2} & \text { if } \lambda_{1} \lambda_{2}>1 \\ \lambda_{1} \geq \lambda_{2} & \text { if } \lambda_{1} \lambda_{2}<1\end{cases}
$$

Indeed, this can be done, if necessary, by using the transformation $\widetilde{x} \mapsto x=Q \widetilde{x}$, where $Q=\left(\begin{array}{cc}0 & 1 \\ 1 & 0\end{array}\right)$; $Q^{-t} B_{1} Q^{-1}$ then switches the order of diagonal entries of $B_{1}$. It is thus enough to prove Theorem 2.1 for the case where $\gamma_{j}=I+\left(B_{j}-I\right) \chi_{D_{j}}, j=1,2$ with $B_{1}$ being a diagonal matrix satisfying (2.3).

Next, assume that $D_{1} \neq D_{2}$, say, $D_{1}$ is not contained in $D_{2}$. Following [17], choose a point $p \in \partial D_{1} \backslash \bar{D}_{2}$, which also lies in the intersection of the convex hull of $D_{1} \cup D_{2}$ and the closure of the connected component of $\Omega \backslash\left(D_{1} \cup D_{2}\right)$ that is in contact with $\partial \Omega$. Consider a disk $B_{r}(p)$ with center $p$ of radius $r>0$ to be specified in a moment. Set $D_{3}=D_{1} \cup B_{r}(p)$. Also, let $D_{4}$ be the union of $D_{1} \cup D_{2}$ and all the components of $\Omega \backslash\left(D_{1} \cup D_{2}\right)$ that do not touch $\partial \Omega$. Finally, let $D_{5}=\Omega \backslash D_{4}$, the connected component of $\Omega \backslash\left(\overline{D_{1} \cup D_{2}}\right)$ whose boundary contains $\partial \Omega$.

Choose the radius $r$ such that $B_{r}(p) \subset \Omega, B_{r}(p) \cap \bar{D}_{2}=\emptyset$ and $\partial D_{4} \cap B_{r}(p)$ is the image of a Lipschitz function $\psi:[0,1] \rightarrow \mathbb{R}^{2}$. Without loss of generality, we may assume $p$ to be the origin $\mathbf{O}$. For the notational simplicity write $B_{3}=B_{1}$, and define $\gamma_{3}$ by

$$
\gamma_{3}=I+\left(B_{3}-I\right) \chi_{D_{3}}\left(=I+\left(B_{1}-I\right) \chi_{D_{3}}\right) .
$$

Recall the following lemma due to Isakov [17].

Lemma 2.2. For $j=2,3$, let $u^{j}$ satisfy $L_{\gamma_{j}} u^{j}=0$ in a neighborhood of $D_{4}$ for $j=2,3$. Then

$$
\int_{D_{1}}\left(B_{1}-I\right) \nabla u^{2} \cdot \nabla u^{3} d y=\int_{D_{2}}\left(B_{2}-I\right) \nabla u^{2} \cdot \nabla u^{3} d y
$$

Notice that the restrictions of $\gamma_{j}$ to $B_{r}(\mathbf{O})$ are the constant matrices. Indeed, they are the identity matrix and the matrix $B_{1}$ for $j=2$ and $j=3$, respectively, which we will denote by $\gamma_{2}^{\prime}$ and $\gamma_{3}^{\prime}$. Then the following lemma is a suitable modification of a result in [15].

Lemma 2.3. Suppose that a function $\widetilde{U}^{j} \in L_{l o c}^{1}\left(\mathbb{R}^{2}\right) \cap H_{l o c}^{1}\left(\mathbb{R}^{2} \backslash\{\mathbf{O}\}\right)$, for $j=2,3$, satisfies

$$
\nabla \cdot\left(\gamma_{j}^{\prime} \nabla \widetilde{U}^{j}\right)=0 \text { in } \mathbb{R}^{2} \backslash \mathbf{O}
$$

Then there exists a family of functions $\left\{\widetilde{u}^{j, \xi}\right\}, \xi \in B_{r / 2}(\mathbf{O}) \backslash \bar{D}_{4}$, such that

$$
\begin{gathered}
\sup _{\xi \in B_{r / 2}(\mathbf{O}) \backslash \bar{D}_{4}}\left\|\widetilde{u}^{j, \xi}(\cdot)-\widetilde{U}^{j}(\cdot-\xi)\right\|_{H^{1}\left(D_{4}\right)}<\infty, \\
\left.\widetilde{u}^{j, \xi}\right|_{\partial \Omega}=0, \\
\nabla \cdot\left(\gamma_{j} \nabla \widetilde{u}^{j, \xi}\right)=0 \text { in } D_{4, \xi}=\left\{y \mid d\left(y, D_{4}\right)<d\left(\xi, D_{4}\right) / 2\right\} .
\end{gathered}
$$


Proof. Let $\xi \in B_{r / 2}(\mathbf{O}) \backslash \bar{D}_{4}$ be arbitrary. First, a mollification argument will provide a sequence of $C^{\infty}(\bar{\Omega})$ functions $\left\{\phi_{m}^{j, \xi}\right\}_{m \geq 1}$ such that

$$
\phi_{m}^{j, \xi}(\cdot) \rightarrow \widetilde{U}^{j}(\cdot-\xi) \text { in } H_{l o c}^{1}(\Omega \backslash\{\xi\}) \quad \text { as } m \rightarrow \infty .
$$

For each $m$, let $w_{m}^{j, \xi}$ and $z_{m}^{j, \xi} \in H^{1}(\Omega)$ be the unique solutions of the following problems:

$$
\begin{aligned}
\nabla \cdot\left(\gamma_{j} \nabla w_{m}^{j, \xi}\right)=\nabla \cdot\left(\gamma_{j}^{\prime} \nabla \phi_{m}^{j, \xi}\right) & \text { in } \Omega, \\
w_{m}^{j, \xi}=0 & \text { on } \partial \Omega,
\end{aligned}
$$

and

$$
\begin{gathered}
\nabla \cdot\left(\gamma_{j}^{\prime} \nabla z_{m}^{j, \xi}\right)=0 \text { in } \Omega, \\
z_{m}^{j, \xi}=-\phi_{m}^{j, \xi} \text { on } \partial \Omega .
\end{gathered}
$$

By elliptic regularity and the trace theorem, we get

$$
\left\|z_{m}^{j, \xi}-z_{m^{\prime}}^{j, \xi}\right\|_{H^{1}(\Omega)} \leq C\left\|\phi_{m}^{j, \xi}-\phi_{m^{\prime}}^{j, \xi}\right\|_{H^{1 / 2}(\partial \Omega)} \leq C\left\|\phi_{m}^{j, \xi}-\phi_{m^{\prime}}^{j, \xi}\right\|_{H^{1}\left(\Omega \backslash B_{r}(\mathbf{O})\right)},
$$

from which we have $z^{j, \xi} \in H^{1}(\Omega)$ such that

$$
\begin{aligned}
& z_{m}^{j, \xi} \rightarrow z^{j, \xi} \text { as } m \rightarrow \infty \text { in } H^{1}(\Omega), \\
& \sup _{\xi \in B_{r / 2}(\mathbf{O}) \backslash \bar{D}_{4}}\left\|z^{j, \xi}\right\|_{H^{1}(\Omega)}<\infty .
\end{aligned}
$$

Setting $v_{m}^{j, \xi}=w_{m}^{j, \xi}-\phi_{m}^{j, \xi}-z_{m}^{j, \xi}$, we see that

$$
\begin{aligned}
\nabla \cdot\left(\gamma_{j} \nabla v_{m}^{j, \xi}\right)=\nabla \cdot\left(\left(\gamma_{j}^{\prime}-\gamma_{j}\right) \nabla\left(\phi_{m}^{j, \xi}+z_{m}^{j, \xi}\right)\right) & \text { in } \Omega \\
v_{m}^{j, \xi}=0 & \text { on } \partial \Omega .
\end{aligned}
$$

Since $\gamma_{j}^{\prime}=\gamma_{j}$ in $B_{r}(\mathbf{O})$, the estimate (2.8) leads to

$$
\begin{aligned}
\left\|v_{m}^{j, \xi}-v_{m^{\prime}}^{j, \xi}\right\|_{H^{1}(\Omega)} & \leq C\left\{\left\|\phi_{m}^{j, \xi}-\phi_{m^{\prime}}^{j, \xi}\right\|_{H^{1}\left(\Omega \backslash B_{r}(\mathbf{O})\right)}+\left\|z_{m}^{j, \xi}-z_{m^{\prime}}^{j, \xi}\right\|_{H^{1}(\Omega) \backslash B_{r}(\mathbf{O})}\right\} \\
& \leq C^{\prime}\left\|\phi_{m}^{j, \xi}-\phi_{m^{\prime}}^{j, \xi}\right\|_{H^{1}\left(\Omega \backslash B_{r}(\mathbf{O})\right)} .
\end{aligned}
$$

From this it follows that, for all $j, \xi$, there is a $v^{j, \xi} \in H^{1}(\Omega)$ such that

$$
\begin{aligned}
& v_{m}^{j, \xi} \rightarrow v^{j, \xi} \text { in } H^{1}(\Omega) \quad \text { as } m \rightarrow \infty, \\
& \sup _{\xi \in B_{r / 2}(\mathbf{O}) \backslash \bar{D}_{4}}\left\|v^{j, \xi}\right\|_{H^{1}(\Omega)}<\infty .
\end{aligned}
$$

For each $j$, let $\widetilde{u}^{j, \xi}=\widetilde{U}^{j}(\cdot-\xi)+v^{j, \xi}+z^{j, \xi}$. It is then immediate to see that $\left\{\widetilde{u}^{j, \xi}\right\}$ satisfies the properties of (2.6). This completes the proof.

Since $\mathbf{O}$ lies in the convex hull of $D_{1} \cup D_{2}$, there is a straight line passing through $\mathbf{O}$ such that $D_{1} \cap B_{r}(\mathbf{O})$ is contained in one side of this straight line. We may assume this straight line to be the $y_{1}$ axis and the $y_{2}$ axis to be a vertical line to this line. Assume further that the $y_{2}$ axis passes through $D_{1} \cap B_{r}(\mathbf{O})$, if necessary, after 
rotating the $y_{1}$ axis. Choose the positive directions of the $y_{2}$ axis toward $D_{1} \cap B_{r}(\mathbf{O})$. Since $D_{1}$ is Lipschitz, there are two cones $K^{i}$ and $K^{e}$ with angles $0 \leq \theta^{i}<\frac{\pi}{2}$ at $\mathbf{O}$ satisfying [13]

$$
B_{r}(\mathbf{O}) \cap \bar{K}^{i} \subset \bar{D}_{1}, \quad B_{r}(\mathbf{O}) \cap \bar{K}^{e} \cap \bar{D}_{1}=\{\mathbf{O}\} .
$$

More explicitly, let us denote such cones by

$$
K^{i}=\left\{\left(y_{1}, y_{2}\right) \in \mathbb{R}^{2} \mid \frac{\pi}{2}-\theta^{i}<\arg \left(y_{1}+i y_{2}\right)<\frac{\pi}{2}+\theta^{i}\right\},
$$

with $K^{e}$ being the reflection of $K^{i}$ to the $y_{1}$-axis. Here and in what follows, the local branch of the argument function $\arg$ is chosen as the negative $y_{2}$ axis. For $j=2,3$, let

$$
\widetilde{u}^{j}=\frac{1}{2} \log \left(y_{1}^{2}+\tau_{j}^{2} y_{2}^{2}\right)
$$

where $\tau_{2}=1, \tau_{3}=\sqrt{\frac{\lambda_{1}}{\lambda_{2}}}$. Then one can verify that the function $\widetilde{u}^{j} \in L_{l o c}^{1}\left(\mathbb{R}^{2}\right) \cap H_{l o c}^{1}\left(\mathbb{R}^{2} \backslash\{\mathbf{O}\}\right)$ satisfies (2.5). Then let $\left\{\widetilde{u}^{j, \xi}\right\}_{\xi \in B_{r / 2}(\mathbf{O}) \backslash \bar{D}_{4}}, j=2,3$, be given such that (2.6) is fulfilled with $\widetilde{u}^{j}$ in place of $\widetilde{U}^{j}$, respectively, as in Lemma 2.3. Recalling from (2.6c) that $D_{4, \xi}$ is a neighborhood of $D_{4}$, we see that the orthogonality relation (2.4) applies to $\widetilde{u}^{j, \xi}, j=2,3$, with $\xi \in K^{e} \cap B_{r / 2}(\mathbf{O})$, which yields

$$
\begin{aligned}
\int_{D_{1} \cap B_{r}(\mathbf{O})} & \left(B_{1}-I\right) \nabla \widetilde{u}^{2, \xi} \cdot \nabla \widetilde{u}^{3, \xi} d y \\
= & \int_{D_{1}}\left(B_{1}-I\right) \nabla \widetilde{u}^{2, \xi} \cdot \nabla \widetilde{u}^{3, \xi} d y-\int_{D_{1} \backslash B_{r}(\mathbf{O})}\left(B_{1}-I\right) \nabla \widetilde{u}^{2, \xi} \cdot \nabla \widetilde{u}^{3, \xi} d y \\
= & \int_{D_{2}}\left(B_{2}-I\right) \nabla \widetilde{u}^{2, \xi} \cdot \nabla \widetilde{u}^{3, \xi} d y-\int_{D_{1} \backslash B_{r}(\mathbf{O})}\left(B_{1}-I\right) \nabla \widetilde{u}^{2, \xi} \cdot \nabla \widetilde{u}^{3, \xi} d y .
\end{aligned}
$$

Notice that the last two integrals are bounded regardless of $\xi \in K^{e} \cap B_{r / 2}(\mathbf{O})$ by (2.6a). Consequently, the integral (2.10) is bounded.

In the meanwhile, we are going to show in the following that the integral (2.10) is unbounded as $\xi \rightarrow \mathbf{O}$, which leads to a contradiction. Decompose (2.10) into the sum of $I_{1}, I_{2}, I_{3}$ and $I_{4}$, where

$$
\begin{aligned}
& I_{1}=\int_{D_{1} \cap B_{r}(\mathbf{O})}\left(B_{1}-I\right) \nabla \widetilde{u}^{2}(y-\xi) \cdot \nabla \widetilde{u}^{3}(y-\xi) d y \\
& I_{2}=\int_{D_{1} \cap B_{r}(\mathbf{O})}\left(B_{1}-I\right) \nabla \cdot\left(\widetilde{u}^{2, \xi}(y)-\widetilde{u}^{2}(y-\xi)\right) \nabla \widetilde{u}^{3}(y-\xi) d y \\
& I_{3}=\int_{D_{1} \cap B_{r}(\mathbf{O})}\left(B_{1}-I\right) \nabla \widetilde{u}^{2}(y-\xi) \cdot \nabla\left(\widetilde{u}^{3, \xi}(y)-\widetilde{u}^{3}(y-\xi)\right) d y \\
& I_{4}=\int_{D_{1} \cap B_{r}(\mathbf{O})}\left(B_{1}-I\right) \nabla\left(\widetilde{u}^{2, \xi}(y)-\widetilde{u}^{2}(y-\xi)\right) \cdot \nabla\left(\widetilde{u}^{3, \xi}(y)-\widetilde{u}^{3}(y-\xi)\right) d y
\end{aligned}
$$

Due to (2.6a), an upper bound of $I_{4}$ is easily obtained, say, $\left|I_{4}\right|<C_{0}$. Next, again by (2.6a) and Young's inequality, there exist $C_{1}>0$ and $C_{2}>0$ such that

$$
\left|I_{2}\right|+\left|I_{3}\right| \leq C_{1} \epsilon \int_{D_{1} \cap B_{r}(\mathbf{O})} \frac{d y}{|y-\xi|^{2}}+\frac{C_{2}}{\epsilon}
$$

for all $\xi \in K^{i} \cap B_{r / 2}(\mathbf{O})$ and $\epsilon>0$. Using the following inequality,

$$
\begin{aligned}
\left|I_{1}+I_{2}+I_{3}+I_{4}\right| & \geq\left|I_{1}\right|-\left|I_{2}\right|-\left|I_{3}\right|-\left|I_{4}\right| \\
& \geq\left|I_{1}\right|-C_{1} \epsilon \int_{D_{1} \cap B_{r}(\mathbf{O})} \frac{d y}{|y-\xi|^{2}}-\frac{C_{2}}{\epsilon}-C_{0},
\end{aligned}
$$


we will show that (2.11a) is unbounded as $\xi \rightarrow \mathbf{O}$.

For $s<r / 2$, choose $\xi_{s} \in K^{e} \cap B_{r / 2}(\mathbf{O})$ such that $\xi_{s}=(0,-s)$. Depending on $s$, denote by $\left(\rho_{s}, \theta_{s}\right)$ the polar coordinate system with origin at $\xi_{s}$ such that $y-\xi_{s}=\left(\rho_{s} \cos \theta_{s}, \rho_{s} \sin \theta_{s}\right)$, where $\rho_{s}(y)=\left|y-\xi_{s}\right|$ and $\theta_{s}(y)=\arg \left(y-\xi_{s}\right)$. Let $M_{s}=\max _{y \in S}\left|y-\xi_{s}\right|$, where $S=\partial D_{1} \cap \partial B_{r}(\mathbf{O})$ consists of a set of two points for sufficiently small $r$. Notice that $M_{s}$ converges to $r$ as $s$ converges to 0 . Take the two points $P_{1}, P_{2} \in \partial K^{i}$ such that $\arg \left(P_{1}\right)=\frac{\pi}{2}-\theta^{i}, \arg \left(P_{2}\right)=\frac{\pi}{2}+\theta^{i}$ and $\left|P_{1}\right|=\left|P_{2}\right|=s$. Then we have $\arg \left(P_{1}-\xi_{s}\right)=\frac{\pi}{2}-\frac{\theta^{i}}{2}$, $\arg \left(P_{2}-\xi_{s}\right)=\frac{\pi}{2}+\frac{\theta^{i}}{2}$ and $\left|P_{1}-\xi_{s}\right|=\left|P_{2}-\xi_{s}\right|=\sqrt{2} s \sqrt{1+\cos \theta^{i}}$. Let us decompose $D_{1} \cap B_{r}(\mathbf{O})$ into the three regions:

$$
\begin{aligned}
E_{s} & =\left\{y \in D_{1} \cap B_{r}(\mathbf{O}) \mid \sqrt{2} s \sqrt{1+\cos \theta^{i}} \leq \rho_{s}(y) \leq M_{s}\right\} \\
F_{s} & =\left\{y \in D_{1} \cap B_{r}(\mathbf{O}) \mid s \leq \rho_{s}(y)<\sqrt{2} s \sqrt{1+\cos \theta^{i}}\right\} \\
G_{s} & =\left\{y \in D_{1} \cap B_{r}(\mathbf{O}) \mid \rho_{s}(y)>M_{s}\right\} .
\end{aligned}
$$

By (2.11) and (2.12), $\left|I_{1}+I_{2}+I_{3}+I_{4}\right|$ is bounded below as follows:

$$
\begin{aligned}
\left|I_{1}+I_{2}+I_{3}+I_{4}\right| \geq & \left|\int_{E_{s}}\left(B_{1}-I\right) \nabla \widetilde{u}^{2}(y-\xi) \cdot \nabla \widetilde{u}^{3}(y-\xi) d y\right|-C_{1} \epsilon \int_{E_{s}} \frac{d y}{|y-\xi|^{2}} \\
& -\left|\int_{F_{s}}\left(B_{1}-I\right) \nabla \widetilde{u}^{2}(y-\xi) \cdot \nabla \widetilde{u}^{3}(y-\xi) d y\right|-C_{1} \epsilon \int_{F_{s}} \frac{d y}{|y-\xi|^{2}} \\
& -\left|\int_{G_{s}}\left(B_{1}-I\right) \nabla \widetilde{u}^{2}(y-\xi) \cdot \nabla \widetilde{u}^{3}(y-\xi) d y\right|-C_{1} \epsilon \int_{G_{s}} \frac{d y}{|y-\xi|^{2}} \\
& -\frac{C_{2}}{\epsilon}-C_{0} .
\end{aligned}
$$

We will show that the integrals over $F_{s}$ and $G_{s}$ are uniformly bounded, while that over $E_{s}$ is unbounded. Since $G_{s} \subset G_{r}$ for all $s \leq r \leq 1$, the integral over $G_{s}$ is bounded uniformly in $s$ as follows:

$$
\begin{aligned}
& \left|\int_{G_{s}}\left(B_{1}-I\right) \nabla \widetilde{u}^{2}(y-\xi) \cdot \nabla \widetilde{u}^{3}(y-\xi) d y\right|+C_{1} \epsilon \int_{G_{s}} \frac{d y}{|y-\xi|^{2}} \\
& \quad \leq \max \left(\left|\lambda_{1}-1\right|,\left|\lambda_{2}-1\right|\right) \int_{G_{r}}\left|\nabla \widetilde{u}^{2}(y-\xi)\right| \cdot\left|\nabla \widetilde{u}^{3}(y-\xi)\right| d y+C_{1} \epsilon \int_{G_{r}} \frac{d y}{|y-\xi|^{2}} \\
& \quad \leq\left(C_{3}+C_{1} \epsilon\right) \frac{\left|G_{r}\right|}{r^{2}} \leq\left(C_{3}+C_{1} \epsilon\right) \pi .
\end{aligned}
$$

Turn to estimate the integrals over $E_{s}$ and $F_{s}$.

$$
\begin{aligned}
\mid \int_{E_{s}}( & \left.B_{1}-I\right) \nabla \widetilde{u}^{2}(y-\xi) \cdot \nabla \widetilde{u}^{3}(y-\xi) d y \mid-C_{1} \epsilon \int_{E_{s}} \frac{d y}{|y-\xi|^{2}} \\
= & \left|\int_{\sqrt{2} s \sqrt{1+\cos \theta^{i}}}^{M_{s}} \frac{1}{\rho_{s}} \int_{\alpha(s)}^{\beta(s)}\left[\frac{\lambda_{1} \lambda_{2}}{\lambda_{2} \cos ^{2} \theta_{s}+\lambda_{1} \sin ^{2} \theta_{s}}-1\right] d \theta_{s} d \rho_{s}\right| \\
& \quad-C_{1} \epsilon \int_{\sqrt{2} s \sqrt{1+\cos \theta^{i}}}^{M_{s}} \frac{1}{\rho_{s}} \int_{\alpha(s)}^{\beta(s)} d \theta_{s} d \rho_{s},
\end{aligned}
$$


and

$$
\begin{gathered}
\left|\int_{F_{s}}\left(B_{1}-I\right) \nabla \widetilde{u}^{2}(y-\xi) \cdot \nabla \widetilde{u}^{3}(y-\xi) d y\right|+C_{1} \epsilon \int_{F_{s}} \frac{d y}{|y-\xi|^{2}} \\
=\left|\int_{s}^{\sqrt{2} s \sqrt{1+\cos \theta^{2}}} \frac{1}{\rho_{s}} \int_{\alpha(s)}^{\beta(s)}\left[\frac{\lambda_{1} \lambda_{2}}{\lambda_{2} \cos ^{2} \theta_{s}+\lambda_{1} \sin ^{2} \theta_{s}}-1\right] d \theta_{s} d \rho_{s}\right| \\
\quad+C_{1} \epsilon \int_{s}^{\sqrt{2} s \sqrt{1+\cos \theta^{2}}} \frac{1}{\rho_{s}} \int_{\alpha(s)}^{\beta(s)} d \theta_{s} d \rho_{s},
\end{gathered}
$$

where

$$
\begin{aligned}
& \alpha(s)=\min _{y \in D_{1} \cap B_{r}(\mathbf{O})} \arg \left(y-\xi_{s}\right), \quad \beta(s)=\max _{y \in D_{1} \cap B_{r}(\mathbf{O})} \arg \left(y-\xi_{s}\right) . \\
& \left|y-\xi_{s}\right|=\rho_{s} \quad\left|y-\xi_{s}\right|=\rho_{s}
\end{aligned}
$$

Notice that

$$
\left|\int_{\alpha}^{\beta}\left[\frac{\lambda_{1} \lambda_{2}}{\lambda_{2} \cos ^{2} \theta+\lambda_{1} \sin ^{2} \theta}-1\right] d \theta\right| \leq(\beta-\alpha)\left[1+\frac{\lambda_{1} \lambda_{2}}{\min \left(\lambda_{1}, \lambda_{2}\right)}\right] \leq \pi\left[1+\frac{\lambda_{1} \lambda_{2}}{\min \left(\lambda_{1}, \lambda_{2}\right)}\right]:=C_{4} .
$$

More explicitly, a change of variables implies, for $0 \leq \alpha<\frac{\pi}{2}<\beta \leq \pi$,

$$
\begin{aligned}
& \int_{\alpha}^{\beta} \frac{\left(\lambda_{1}-1\right) \lambda_{2} \cos ^{2} \theta+\lambda_{1}\left(\lambda_{2}-1\right) \sin ^{2} \theta}{\lambda_{2} \cos ^{2} \theta+\lambda_{1} \sin ^{2} \theta} d \theta \\
& =\sqrt{\lambda_{1} \lambda_{2}}\left(\pi-\arctan \left(\sqrt{\frac{\lambda_{1}}{\lambda_{2}}} \tan (\pi-\beta)\right)-\arctan \left(\sqrt{\frac{\lambda_{1}}{\lambda_{2}}} \tan \alpha\right)\right)-(\beta-\alpha),
\end{aligned}
$$

where the range of $\arctan$ is contained in $\left(-\frac{\pi}{2}, \frac{\pi}{2}\right)$. Define the continuous functions $\widetilde{\delta}:\left[0, \frac{\pi}{2}\right] \times\left[\frac{\pi}{2}, \pi\right] \rightarrow \mathbb{R}$, and $\delta:[0, r] \rightarrow \mathbb{R}$ by

$$
\widetilde{\delta}(\alpha, \beta)= \begin{cases}\sqrt{\lambda_{1} \lambda_{2}}\left(\pi-\arctan \left(\sqrt{\frac{\lambda_{1}}{\lambda_{2}}} \tan (\pi-\beta)\right)-\arctan \left(\sqrt{\frac{\lambda_{1}}{\lambda_{2}}} \tan \alpha\right)\right)-(\beta-\alpha), & 0 \leq \alpha<\frac{\pi}{2}<\beta \leq \pi, \\ \sqrt{\lambda_{1} \lambda_{2}}\left(\pi-\arctan \left(\sqrt{\frac{\lambda_{1}}{\lambda_{2}}} \tan (\pi-\beta)\right)-\frac{\pi}{2}\right)-\left(\beta-\frac{\pi}{2}\right), & \alpha=\frac{\pi}{2}, \frac{\pi}{2}<\beta \leq \pi, \\ \sqrt{\lambda_{1} \lambda_{2}}\left(\pi-\frac{\pi}{2}-\arctan \left(\sqrt{\frac{\lambda_{1}}{\lambda_{2}}} \tan \alpha\right)\right)-\left(\frac{\pi}{2}-\alpha\right), & 0 \leq \alpha<\frac{\pi}{2}, \beta=\frac{\pi}{2},\end{cases}
$$

and

$$
\delta(s)=\widetilde{\delta}(\alpha(s), \beta(s)) .
$$

Using the constant $C_{4}$ in (2.17), an upper bound of the integral (2.16) is obtained:

$$
\begin{gathered}
\left|\int_{s}^{\sqrt{2} s \sqrt{1+\cos \theta^{i}}} \frac{1}{\rho_{s}} \delta\left(\rho_{s}\right) d \rho_{s}\right|+C_{1} \epsilon \int_{s}^{\sqrt{2} s \sqrt{1+\cos \theta^{i}}} \frac{1}{\rho_{s}} d \rho_{s} \\
\leq\left(C_{4}+C_{1} \epsilon\right) \log \left(\sqrt{2} \sqrt{1+\cos \theta^{i}}\right) .
\end{gathered}
$$

Finally, let us consider the integral (2.15). If $\lambda_{1} \lambda_{2}>1$, by (2.3),

$$
\arctan \left(\sqrt{\frac{\lambda_{1}}{\lambda_{2}}} \tan (\pi-\beta)\right)<\pi-\beta \text { and } \arctan \left(\sqrt{\frac{\lambda_{1}}{\lambda_{2}}} \tan \alpha\right)<\alpha,
$$


resulting in

$$
\delta(s) \geq\left(\lambda_{1} \lambda_{2}-1\right)(\beta(s)-\alpha(s))>0 .
$$

On the other hand if $\lambda_{1} \lambda_{2}<1$, again by (2.3),

$$
\arctan \left(\sqrt{\frac{\lambda_{1}}{\lambda_{2}}} \tan (\pi-\beta)\right)>\pi-\beta \text { and } \arctan \left(\sqrt{\frac{\lambda_{1}}{\lambda_{2}}} \tan \alpha\right)>\alpha,
$$

which leads to

$$
\delta(s) \leq\left(\lambda_{1} \lambda_{2}-1\right)(\beta(s)-\alpha(s))<0 .
$$

Therefore, as $s$ varies, $\delta(s)$ does not change its sign for fixed $\lambda_{1}, \lambda_{2}$. In $E_{s}$, it is obvious that $0 \leq \alpha(s) \leq \frac{\pi}{2}-\frac{\theta^{i}}{2}$ and $\frac{\pi}{2}+\frac{\theta^{i}}{2} \leq \beta(s) \leq \pi$, a lower bound of $|\delta(s)|$ being obtained:

$$
|\delta(s)| \geq\left|\lambda_{1} \lambda_{2}-1\right| \theta^{i}>0 .
$$

The choice of $\epsilon=\frac{\left|\lambda_{1} \lambda_{2}-1\right|}{2 C_{1}}$ gives a lower bound of the integral (2.15) over $E_{s}$ :

$$
\begin{aligned}
& \left|\int_{E_{s}}\left(B_{1}-I\right) \nabla \widetilde{u}^{2}(y-\xi) \cdot \nabla \widetilde{u}^{3}(y-\xi) d y\right|-C_{1} \epsilon \int_{E_{s}} \frac{d y}{|y-\xi|^{2}} \\
& \quad \geq \int_{\sqrt{2} s \sqrt{1+\cos \theta^{i}}}^{M_{s}} \frac{1}{\rho_{s}}\left[\left|\lambda_{1} \lambda_{2}-1\right|-C_{1} \epsilon\right] d \rho_{s} \\
& \quad \geq \frac{\left|\lambda_{1} \lambda_{2}-1\right|}{2} \int_{\sqrt{2} s \sqrt{1+\cos \theta^{i}}}^{M_{s}} \frac{1}{\rho_{s}} d \rho_{s},
\end{aligned}
$$

Summing up (2.13), (2.14), (2.18) and (2.19), we get

$$
\begin{aligned}
\left|I_{1}+I_{2}+I_{3}+I_{4}\right| \geq & \frac{\left|\lambda_{1} \lambda_{2}-1\right|}{2} \int_{\sqrt{2} s \sqrt{1+\cos \theta^{2}}}^{M_{s}} \frac{1}{\rho} d \rho-\left(C_{4}+\frac{\left|\lambda_{1} \lambda_{2}-1\right|}{2}\right) \log \left(\sqrt{2\left(1+\cos \theta^{i}\right)}\right) \\
& -\left(C_{3}+\frac{\left|\lambda_{1} \lambda_{2}-1\right|}{2}\right) \pi-\frac{2 C_{2}}{\left|\lambda_{1} \lambda_{2}-1\right|}-C_{0} .
\end{aligned}
$$

All terms in the right side of (2.20) are constant except the first one that tends to $\frac{\left|\lambda_{1} \lambda_{2}-1\right|}{2} \int_{0}^{r} \frac{1}{\rho} d \rho$ to blow up. Hence $I_{1}+I_{2}+I_{3}+I_{4}$ becomes unbounded as $\xi_{s}$ approaches to $\mathbf{O}$, which is a contradiction originated from the assumption that $D_{1} \neq D_{2}$. This proves that $D_{1}=D_{2}$.

\subsection{The Proof of Theorem 2.1: $B_{1}=B_{2}$}

It remains to prove $B_{1}=B_{2}$. Let $D=D_{1}=D_{2}$ and take $\hat{f}^{\eta}(\xi)=\eta \cdot \xi$, for a fixed vector $\eta \in \mathbb{R}^{2}$. Then by the uniqueness of the Dirichlet problem, $\eta \cdot \xi$ is the unique solution of

$$
\begin{array}{rlll}
\nabla \cdot\left(B_{1} \nabla \hat{v}\right) & =0 \quad \text { in } & D, \\
\hat{v} & =\hat{f}^{\eta} \text { on } & \partial D .
\end{array}
$$

Next take a harmonic function $v$ in $\Omega \backslash \bar{D}$ such that

$$
\begin{array}{rlr}
v^{\eta} & :=\hat{v}^{\eta}=\hat{f}^{\eta} & \text { on } \partial D, \\
\frac{\partial v^{\eta}}{\partial \nu} & :=\nu \cdot B_{1} \nabla \hat{v}^{\eta} & \text { on } \partial D .
\end{array}
$$


Let $f^{\eta}$ be the trace on $\partial \Omega$ of $v^{\eta} \in H^{1}(\Omega \backslash \bar{D})$ and $u_{j}^{\eta}$ be the solution of (1.1) replacing $f$ by $f^{\eta}$ for $j=1,2$, respectively. By uniqueness, $u_{1}^{\eta}(\xi)=v^{\eta}(\xi) \chi_{\Omega \backslash \bar{D}}(\xi)+\eta \cdot \xi \chi_{D}(\xi)$. Suppose that the Dirichlet-to-Neumann maps $\Lambda_{\gamma}$ are identical corresponding to $\gamma=I+\left(B_{1}-I\right) \chi_{D}$ and $\gamma=I+\left(B_{2}-I\right) \chi_{D}$. Then $u_{1}^{\eta}=u_{2}^{\eta}$ in $\Omega \backslash \bar{D}$ by unique continuation. Thus the transmission condition implies that $u_{2}^{\eta}$ satisfies

$$
\begin{aligned}
\nabla \cdot\left(B_{2} \nabla u_{2}^{\eta}\right) & =0 \quad \text { in } \quad D, \\
u_{2}^{\eta} & =\hat{f}^{\eta} \quad \text { on } \quad \partial D,
\end{aligned}
$$

and hence $u_{2}^{\eta}(\xi)=\eta \cdot \xi$ in $D$. Moreover,

$$
\nu\left(B_{1}-B_{2}\right) \eta=\frac{\partial u_{1}^{\eta}}{\partial \nu^{+}}-\frac{\partial u_{2}^{\eta}}{\partial \nu^{+}}=0
$$

on $\partial D$ for all $\eta \in \mathbb{R}^{2}$. Consequently, get $B_{1}=B_{2}$. Therefore we prove Theorem 2.1.

\section{Anisotropic Inverse Scattering Problem}

In this section we apply the analysis carried out for the inverse conductivity problem (1.1) to the inverse scattering problem (1.2). As the argument of the proof is identical to that in Section 2, it will be briefly sketched.

\subsection{Uniqueness Result}

Analogously to the conductivity case, we consider the case

$$
\gamma=A+(B-A) \chi_{D} \text { and } q=1+(\rho-1) \chi_{D},
$$

where $A$ is a known symmetric positive-definite matrix, $B$ a positive-definite matrix, and $D$ a Lipschitz domain compactly embedded in $\mathbb{R}^{2}$ such that $\mathbb{R}^{2} \backslash D$ is connected. Moreover, we assume that the matrices $A$ and $B$ are constant.

The uniqueness of our inverse scattering problem is stated in the following theorem.

Theorem 3.1. Let $\gamma_{j}=A+\left(B_{j}-A\right) \chi_{D_{j}}, q_{j}=1+\left(\rho_{j}-1\right) \chi_{D_{j}}$ and $u_{j}$ be the solution of (1.2) for $\gamma=\gamma_{j}$ and $q=q_{j}$ for $j=1,2$. Assume $\operatorname{det}\left(B_{j}\right) \neq \operatorname{det}(A), j=1,2$. Then

$$
u_{\infty}^{1}(\cdot ; d)=u_{\infty}^{2}(\cdot ; d) \text { for all direction } d \in S^{1}
$$

implies

$$
D_{1}=D_{2}, B_{1}=B_{2} \text { and } \rho_{1}=\rho_{2} .
$$

\subsection{Proof of Theorem 3.1: a sketch}

First of all, inverse conductivity and scattering problems are closely related with the following approximation property Lemmale:consca, which can be found in [18]. 
Lemma 3.2. Let $\Omega$ be an open set where $\mathbb{R}^{2} \backslash \bar{\Omega}$ is connected, $\bar{D} \in \Omega$ be a Lipschitz domain such that $\Omega \backslash \bar{D}$ is connected and $v$ be a solution to the equation

$$
\nabla \cdot(\gamma \nabla v)+k^{2} q v=0 \text { in } \Omega .
$$

Then $v$ is the $H^{1}(D)$-limit of linear combination of solutions $u(\cdot: d), d \in S^{1}$ satisfying (1.2).

With the aid of this lemma we can apply the analysis carried out for our inverse conductivity problem in the previous section to analyze our inverse scattering problem.

We then follow the arguments in Section 2 to prove Theorem 3.1. The same changes of variables convert $A, B_{1}, B_{2}$ into $I$, a diagonal matrix, and a symmetric positive-definite matrix, respectively. Let $D_{3}, D_{4}, D_{5}$ and $p$ be identical to those appeared in the proof of Theorem 2.1. Analogously to Lemma 2.2, the following lemma holds: (See also [18])

Lemma 3.3. If (3.21) holds, then

$$
\int_{D_{1}}\left(-\left(B_{1}-I\right) \nabla u^{2} \cdot \nabla u^{3}+k^{2} q_{1} u^{3} u^{2}\right) d x=\int_{D_{2}}\left(-\left(B_{2}-I\right) \nabla u^{2} \cdot \nabla u^{3}+k^{2} q_{2} u^{3} u^{2}\right) d x
$$

for all $u_{j}, j=2,3$, such that

$$
\nabla \cdot\left(\gamma_{j} \nabla u^{j}\right)+k^{2} q_{j} u^{j}=0 \quad j=2,3
$$

in some neighborhood of $D_{4}$.

Similarly to Lemma 2.3 , we also have

Lemma 3.4. Suppose that $u^{j}, j=2,3$ satisfy

$$
u^{j} \in L_{l o c}^{1}(\Omega) \cap H_{l o c}^{1}(\Omega \backslash\{a\}) \text { and } \nabla \cdot\left(\gamma_{j}^{\prime} \nabla u^{j}\right)=0 \text { in } D_{4} .
$$

Then there exist $\left\{u^{j, x}\right\}_{x \in B_{r / 2}}$ such that

$$
\sup _{x \in B(r / 2)}\left\|u^{j, x}(\cdot)-u^{j}(\cdot-x)\right\|_{H^{1}\left(D_{4}\right)}<\infty ;\left.u^{j, x}\right|_{\partial \Omega}=0
$$

and

$$
\nabla \cdot \gamma_{j} \nabla u^{j, x}+k^{2} q_{j} u^{j, x}=0 \text { in } D_{4, x}=\left\{y \mid d\left(y, \bar{D}_{4}\right)<d\left(x, \bar{D}_{4}\right) / 2\right\} .
$$

Proof. The proof is identical to that of Lemma 2.3 except (2.7) and (2.9). Take $\phi_{m}^{j, x}, z_{m}^{j, x}$ in the same manner as in Lemma 2.3. Let $w_{m}^{j, x} \in H^{1}(\Omega)$ be such that

$$
\begin{array}{r}
\nabla \cdot\left(\gamma_{j} \nabla w_{m}^{j, x}\right)+k^{2} q w_{m}^{j, x}=\nabla \cdot\left(\gamma_{j}^{\prime} \nabla u_{m}^{j, x}\right) \text { in } \Omega, \\
w_{m}^{j, x}=0 \text { on } \partial \Omega,
\end{array}
$$

and $v_{m}^{j, x}=w_{m}^{j, x}-\phi_{m}^{j, x}-z_{m}^{j, x}$. Then $v_{m}^{j, x}$ satisfies

$$
\begin{array}{r}
\nabla \cdot\left(\gamma_{j} \nabla v_{m}^{j, x}\right)+k^{2} q v_{m}^{j, x}=\nabla \cdot\left(\left(B_{j}^{\prime}-B_{j} \chi_{D_{j}}\right) \nabla\left(\phi_{m}^{j, x}+z_{m}^{j, x}\right)\right) \text { in } \Omega \\
v_{m}^{j, x}=0 \text { on } \partial \Omega .
\end{array}
$$

The rest of the proof follows by the same argument as in Lemma 2.3. 
Choose $u_{j}$ in the same fashion as in the proof of Theorem 2.1, and follow the arguments of Section 2. Then the uniqueness $D_{1}=D_{2}$ and $B_{1}=B_{2}$ is obtained. The uniqueness of $\rho$ follows from the argument given in $[5,18,30]$.

\section{Acknowledgments}

The authors are supported in part by KOSEF 2000-1-10300-001-5, R01-2001-00040200, and KRF-2000015-DS0004.

\section{References}

[1] G. Alessandrini. Stable determination of conductivity by boundary measurements. Applic. Anal., 27:153$172,1988$.

[2] G. Alessandrini. Singular solutions of elliptic equations and the determination of conductivity by boundary measurements. J. Differential Equations, 84:252-272, 1990.

[3] G. Alessandrini and R. Gaburro. Determining conductivity with special anisotropy by boundary measurements. SIAM J. Math. Anal., 33:153-171, 2001.

[4] B. Barcelo, E. Fabes, and J. K. Seo. The inverse conductivity problem with one measurement: Uniqueness for convex polyhedra. Proceedings of AMS, 122:183-189, 1994.

[5] R. M. Brown and G. Uhlmann. Uniqueness in the inverse conductivity problem for non smooth conductivities in two dimensions. Comm. P.D.Es., 22:1009-1027, 1997.

[6] A. P. Calderon. On an inverse boundary value problem. In Seminar on Numerical Analysis and Its Applications to Continuum Physics, pages 65-73, Rio de Janeiro, 1980. Soc. Brasilia de mathematica.

[7] D. Colton and R. Kress. Integral equation methods in scattering Theory. John Wiley \& Sons, 1983.

[8] D. Colton and R. Kress. Inverse acoustic and electromagnetic scattering theory. Springer-Verlag, 1992.

[9] D. Colton and R. Potthast. The inverse electromagnetic scattering problem for an anisotropic medium. Quarterly Journal of Applied Math., 52:349 - 372, 1999.

[10] A. B. de Monvel and D. Shepelsky. Inverse scattering problem for anisotropic media. J Math. Phys., 36:3443-3453, 1995.

[11] G. Eskin. Inverse scattering problem in anisotropic media. Comm. Math. Phys., 199:471-491, 1998.

[12] A. Friedman and V. Isakov. On the uniqueness in the inverse conductivity problem with one measurement. Indiana Univ. Math. J., 38:553-580, 1989.

[13] P. Grisvard. Elliptic problems in non smooth domains. Pitman, 1985.

[14] F. Gylys-Colwell. An inverse problem for the Helmholtz equation. Inverse Problems, 12:139-156, 1996. 
[15] M. Ikehata. Uniqueness of recovery of a discontinuous Lamé parameters. to appear in Journal of inverse and ill-posed problems.

[16] M. Ikehata. Identification of the curve of discontinuity of the determinant of the anisotropic conductivity. J. Inverse and Ill-Posed Problems, 8:273-285, 2000.

[17] V. Isakov. On uniqueness of recovery of a discontinuous conductivity coefficient. Comm. Pure Appl. Math., 41:865-877, 1988.

[18] V. Isakov. On uniqueness in the inverse transmission scattering problem. Comm. P.D.Es., 15:1565-1587, 1990.

[19] V. Isakov. Inverse problems for partial differential equations. Springer Verlag, 1998.

[20] H. Kang and J. K. Seo. The layer potential technique for the inverse conductivity problem. Inverse Problems, 12:267-278, 1996.

[21] H. Kang, J. K. Seo, and D. Sheen. Numerical identification of discontinuous conductivity coefficients. Inverse Problems, 13:113-123, 1997.

[22] H. Ki and D. Sheen. Numerical inversion of discontinuous conductivities. Inverse Problems, 14:33-47, 2000.

[23] A. Kirsh. An Introduction to the mathematical theory of inverse Problems. Springer Verlag, 1996.

[24] A. Kirsh and R. Kress. Uniqueness in inverse obstacle scattering. Inverse Problems, 9:285-299, 1993.

[25] R. Kohn and M. Vogelius. Identification of an unknown conductivity by means of measurement at the boundary. In SIAM-AMS proceedings, volume 14, pages 113-123, 1984.

[26] L. D. Landau and E. M. Lifshitz. Electrodynamics of continuous media. Pergamon Press, 1984.

[27] J. M. Lee and G. Uhlmann. Determining anisotropic real-analytic conductivities by boundary measurements. Comm. Pure Appl. Math., 102:1097-1112, 1989.

[28] W. R. B. Lionheart. Conformal uniqueness results in anisotropic electrical impedance imaging. Inverse Problems, 13:125-134, 1997.

[29] A. I. Nachman. Reconstructions from boundary measurements. Ann. Math., 128:531-576, 1988.

[30] A. I. Nachman. Global uniqueness for a two-dimensional inverse boundary problem. Ann. Math., 142:7196, 1995.

[31] M. Piana. On uniqueness for anisotropic inhomogeneous inverse scattering problems. Inverse Problems, 14:1565-1579, 1998.

[32] R. Potthast. On a concept of uniqueness in inverse scattering for a finite number of incident waves. SIAM J. Appl. Math., 58:666-82, 1998. 
[33] R. Potthast. Electromagnetic scattering from an orthotropic medium. Journal of Integral Equations and Application, 11:197 - 215, 1999.

[34] D. Sheen and D. Shepelsky. Inverse scattering problem for a stratified anisotropic slab. Inverse problems, 15:499-514, 1999.

[35] Z. Sun and G. Uhlmann. Recovery of singularities for formally determined inverse problems. Comm. Math. Phys., 153:431-445, 1993.

[36] J. Sylvester. An anisotropic inverse boundary value problem. Comm. Pure Appl. Math., 103:201-232, 1990.

[37] J. Sylvester and G. Uhlmann. A global uniqueness theorem for an inverse boundary value problem. Ann. Math., 125:153-169, 1987.

[38] J. Sylvester and G. Uhlmann. Inverse boundary value problem at the boundary - continuous dependence. Comm. Pure Appl. Math., 41:197-219, 1988.

[39] J. Sylvester and G. Uhlmann. The Dirichlet to Neumann map and applications, pages 101-139. SIAM, 1990. 